\title{
IV Thrombolysis-Bridging and Endovascular Treatment for Occlusive Internal Carotid Artery Dissection with Tandem Occlusion
}

\author{
Filip Scheperjans ${ }^{a} \quad$ Johanna Pekkola $^{\mathrm{b}}$ Satu Mustanoja ${ }^{a}$ \\ Jukka Putaala $^{\mathrm{a}}$ Marjaana Tiainen $^{\mathrm{a}}$ Leena Ollila $^{\mathrm{a}}$ \\ Tapio Paananen $^{\mathrm{b}}$ Kimmo Lappalainen ${ }^{\mathrm{b}}$ \\ ${ }^{a}$ Department of Neurology, Helsinki University Central Hospital, and \\ Department of Neurological Sciences, University of Helsinki and \\ ${ }^{b}$ Department of Radiology, University of Helsinki and HUS Radiology, Medical \\ Imaging Center, Helsinki, Finland
}

\section{Key Words}

Carotid artery dissection · Carotid stenting · Thrombolysis · Ischemic stroke · Thrombectomy

\begin{abstract}
Compared to other etiologies of ischemic stroke, occlusive internal carotid artery dissection responds worse to intravenous (IV) thrombolysis. Intracranial tandem occlusion is a predictor of poor outcome. A direct endovascular approach has been proposed as a safe and probably superior alternative to IV thrombolysis. However, it may lead to considerable treatment delays. We used rapidly initiated IV thrombolysis-bridging and subsequent endovascular treatment in two patients with severe hemispheric ischemia due to occlusive internal carotid artery dissection with tandem occlusion and achieved good outcomes. Minimizing recanalization times likely improves patient outcome and IV thrombolysisbridging may be a reasonable strategy to achieve this. The positive initial results obtained with endovascular approaches and IV thrombolysis-bridging in this patient group deserve further scientific exploration.
\end{abstract}

\section{Introduction}

Compared to other etiologies of ischemic stroke, occlusive internal carotid artery dissection (ICAD) responds worse to intravenous (IV) thrombolysis [1, 2]. Tandem 
occlusion of the ipsilateral middle cerebral artery (MCA) is a predictor of poor outcome [3]. A direct endovascular approach involving stenting of the internal carotid artery (ICA) and intra-arterial treatment of the intracranial occlusion has been proposed as a safe and probably superior alternative to IV thrombolysis [4]. However, also negative results have been published [5] and the small number of less than 40 reported cases [6] does not allow definitive conclusions. A direct endovascular approach may lead to considerable treatment delays [4] which may negatively affect outcome [7]. We used rapidly initiated IV thrombolysis-bridging and subsequent endovascular treatment in two patients with ICAD and a tandem occlusion.

\section{Cases}

Patient 1 was a previously healthy 32-year-old woman presenting with acute onset right hemiparesis and aphasia (NIH Stroke Scale [NIHSS] score 23). CT perfusion imaging (CTP) and CT angiography (CTA) showed a large ischemic lesion in the left MCA territory caused by occlusive ICAD and tandem M2 occlusion (fig. $1 \mathrm{~A}-\mathrm{C}$ ). IV thrombolysis treatment was given per standard protocol starting at $49 \mathrm{~min}$ after symptom onset and the patient was immediately transferred to the angiography suite. Full dose IV thrombolysis had been administered before the onset of the endovascular procedure at $130 \mathrm{~min}$. After application of 4,500 units of IV heparin, the ICAD was recanalized using two partially overlapping self-expanding nitinol stents (Protege ${ }^{\circledR} 7 \times 40$ and $8 \times 30$ $\mathrm{mm}$, ev3; fig. 1D). Subsequent angiography at $3 \mathrm{~h}$ from symptom onset showed complete recanalization also of the M2 segment (fig. 1E). Clopidogrel was initiated immediately after the procedure. After $24 \mathrm{~h}$ the patient's NIHSS score had decreased to 3 and MRI showed cortical infarction in the left temporal and parietal lobes without hemorrhage or expansion (fig. 1F).

Patient 2 was a previously healthy 52-year-old man with acute onset right hemiparesis and aphasia (NIHSS score 20). CTP showed an ischemic lesion covering the whole left MCA territory (fig. 1A). CTA revealed an intramural hematoma and narrowing of the left ICA with flame-shaped tapering at the skull base consistent with ICAD and tandem M1 occlusion (fig. 1B, C). No contrast filling was visible in the ICA distal to the skull base which is, however, not specific for complete occlusion in the presence of a major intracranial occlusion and slow flow (fig. 1B). IV thrombolysis per standard protocol was initiated at $94 \mathrm{~min}$ after symptom onset. Onset of the endovascular procedure was at 120 min and IV thrombolysis was continued during the beginning of the procedure up to full dose. The first angiogram showed a proximal flame-shaped ICA occlusion at the C2 level and no intracranial flow (fig. 1D). Exploration with a microcatheter confirmed blind ending of the dissection, with access to true lumen after probing with a microwire. It was apparent that the ICAD had progressed during the time between the two examinations (fig. 1B, D). After application of 5,000 units of heparin and stenting of the ICAD (Protege ${ }^{\circledR} 7 \times 40 \mathrm{~mm}$, ev3) angiography showed distal ICA occlusion (fig. 1E). Complete recanalization of the ICA and MCA was achieved by 2 passes with the Trevo ${ }^{\mathrm{TM}}$ device (Concentric Medical) at 5:15 h after symptom onset (fig. 1F). At $24 \mathrm{~h}$ the patient's NIHSS score was 4, CT showed striatocapsular infarction (fig. 1G) and clopidogrel medication was started.

At 9 months both patients were independent, had no relevant motor deficits and were able to drive a car. However, they still had slight neuropsychological difficulties and moderate dysphasia. No procedure-related complications were observed.

\section{Discussion}

Both our patients were at high risk of developing malignant MCA infarction but after the procedures eventually had good outcomes. Although in occlusive ICAD with tandem occlusion IV thrombolysis may sometimes achieve intracranial recanalization [8], hemodynamic compromise and high risk of reocclusion negatively affect outcome if the 
dissection itself is left untreated [1]. If treated with IV thrombolysis only, prognosis of such cases is usually poor $[1,4]$.

Direct endovascular treatment provides high recanalization rates and repair of the dissection. While this approach appears to be safe and superior to IV thrombolysis, it is associated with an average delay of close to $5 \mathrm{~h}$ from symptom onset to treatment of the intracranial occlusion [4]. As a shorter time to intracranial recanalization is associated with a favourable outcome [7], an optimal treatment strategy should minimize the time to recanalization and repair the dissected artery to prevent hemodynamic insufficiency and intracranial reocclusion. If sufficient collaterals exist, a thrombolytic agent given IV reaches the intracranial thrombus and this can be considered as the onset of tandem occlusion treatment [8]. In our patients, IV thrombolysis was initiated at 49 and 94 min after symptom onset, respectively. Such short treatment delays can hardly be achieved with a primary endovascular approach. If the intracranial vessel is recanalized by the IV thrombolytic, perfusion is restored at least to some degree even if the ICA is still occluded. In patient 1 the intracranial occlusion was already recanalized on the post-stenting angiogram at $3 \mathrm{~h}$ from symptom onset without further intervention which may have contributed to the good outcome (fig. 1E). Even if preceding IV thrombolysis treatment does not achieve recanalization, it may facilitate subsequent thrombectomy and shorten the time to recanalization [9].

Collateral circulation has been increasingly recognized as an important factor influencing outcome of patients with ischemic stroke [10]. When considering invasive approaches in stroke treatment it is important to estimate the ratio of infarcted and salvageable tissue to avoid futile recanalizations and hemorrhagic complications. While angiographic studies can visualize the extent of collateral vascularization, only tissue perfusion studies can assess the effectiveness of collateral flow in the micro- and macrocirculation [10]. The presence of a large CTP mismatch ratio is a prerequisite for achieving a favourable clinical outcome. The probability of a favourable outcome in such patients is further independently influenced by concurrence of good collateral status [10] particularly in later time windows ( $>5 \mathrm{~h}$ from onset) [11]. In both of our patients CTP studies demonstrated a large mismatch ratio (fig. 1A). When graded based on CTA images according to the method proposed by Miteff et al. [12] the collateral status was moderate in patient 2 (fig. 1C). In patient 1 the intracranial occlusion was subtotal with marginal flow around the thrombus and therefore the above-mentioned criteria cannot be applied (fig. 1C). These factors may have contributed to the good outcomes.

We found only 3 studies [13-15] in which IV thrombolysis was used in patients before emergency stenting of ICAD, but 2 of these studies included also patients with atherothrombotic occlusions. Only Lekoubou et al. reported detailed procedural and outcome data specifically for 3 ICAD patients treated with a protocol comparable to ours [13]. However, their patients' symptoms were less severe (NIHSS score 6-16). All patients were independent at one month with no procedure-related complications.

Our patients and those reported by Lekoubou et al. [13] were younger (mean age 35 years) than the average stroke patient and it could be argued that this may have contributed to the good outcome. However, our and Lekoubou's [13] ICAD patients were not extraordinarily young since in the study of Engelter et al., $25 \%$ of thrombolysed ICAD patients were younger than 42 years [1]. Although significantly 
younger, ICAD patients do not recover as well after IV thrombolysis as do non-ICAD stroke patients $[1,2]$.

In a systematic review Pham et al. [6] found 140 reported cases of ICAD stenting of which $28 \%$ were performed for acute stroke. The overall technical success rate was $99 \%$ and the procedural complication rate was $1.3 \%$. However, the largest series included only 26 cases and publication bias may be a confounder. The possibility of severe complications must not be underestimated and specifically in the endovascular treatment of ICAD all attempts to find and verify the access to the true lumen should be very careful to avoid distal propagation of the dissection by manipulating the false lumen.

In conclusion, in occlusive ICAD with tandem occlusion endovascular treatment approaches offer high recanalization rates and are probably superior to IV thrombolysis although procedure-related complications are possible [6]. Minimizing recanalization times likely improves patient outcome [7] and IV thrombolysis-bridging may be a reasonable strategy to achieve this $[8,9]$. Selection of suitable patients is crucial and preinterventional demonstration of a significant perfusion mismatch is mandatory [10]. Evaluation of collateral status may become a further tool for patient selection particularly in later time windows, but this method needs further validation $[10,11]$. The positive initial results obtained with endovascular approaches and IV thrombolysis-bridging in this patient group deserve further scientific exploration. 


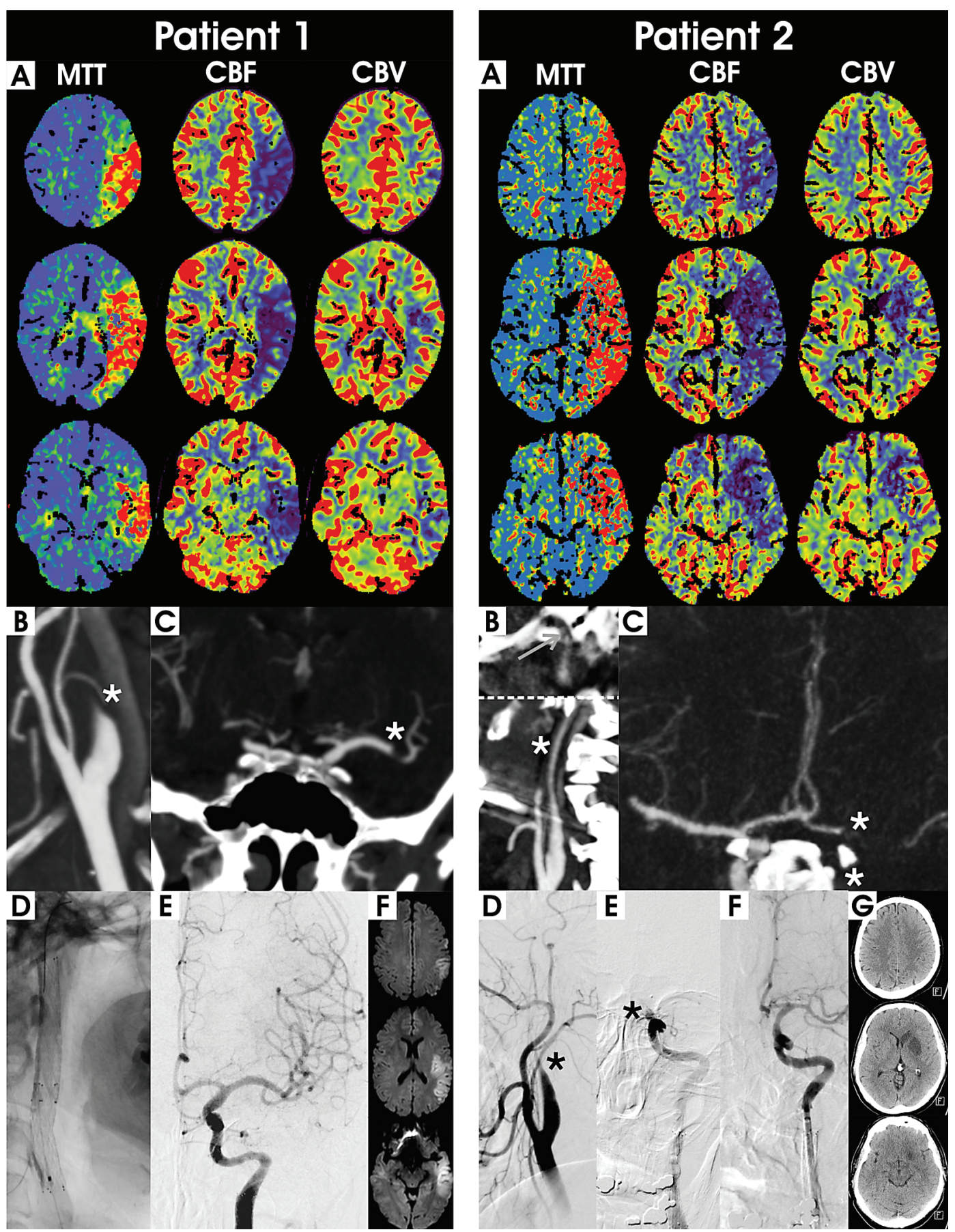


Fig. 1. Patient 1. A CT perfusion maps showing a large hypoperfusion area in the left hemisphere with prolonged mean transit time (MTT) and decreased cerebral blood flow (CBF). A reduction of cerebral blood volume (CBV) suggestive of core infarction is visible in the temporal lobe. A large mismatch between the MTT and CBF maps in relation to the CBV maps suggests a large penumbra. B CTA demonstrating flame-shaped occlusion of the left ICA (asterisk). C CTA of intracranial arteries showing intracranial occlusion of the M2 segment (asterisk). D X-ray image showing the two overlapping stents implanted into the left ICA. E Digital subtraction angiogram (DSA) after ICA stent implantation demonstrating complete recanalization. F Diffusion-weighted MRI at $24 \mathrm{~h}$ showing temporoparietal cortical infarction. Patient 2. A CT perfusion maps showing a large area of hypoperfusion in the left hemisphere with prolonged MTT and decreased CBF. A reduction of CBV suggestive of core infarction is visible in the striatocapsular region. B Two CTA images (separated by dashed line) demonstrating narrowing of the cervical ICA (asterisk) with no contrast filling above the skull base (arrow). The findings are suggestive of dissection and were confirmed by subsequent DSA C CTA of intracranial arteries showing intracranial ICA and M1 occlusion (asterisks). D DSA showing proximal flame-shaped occlusion of the ICA (asterisk). E DSA after ICA stent implantation with persistent distal ICA occlusion (asterisk). F DSA after mechanical thrombectomy demonstrating complete recanalization. G CT at 24 h showing striatocapsular infarction.

\section{References}

-1 Engelter ST, Rutgers MP, Hatz F, Georgiadis D, Fluri F, Sekoranja L, Schwegler G, Müller F, Weder B, Sarikaya H, Lüthy R, Arnold M, Nedeltchev K, Reichhart M, Mattle HP, Tettenborn B, Hungerbühler HJ, Sztajzel R, Baumgartner RW, Michel P, Lyrer PA: Intravenous thrombolysis in stroke attributable to cervical artery dissection. Stroke 2009;40:3772-3776.

2 Qureshi AI, Chaudhry SA, Hassan AE, Zacharatos H, Rodriguez GJ, Suri MF, Lakshminarayan K, Ezzeddine MA: Thrombolytic treatment of patients with acute ischemic stroke related to underlying arterial dissection in the United States. Arch Neurol 2011;68:1536-1542.

- 3 Rubiera M, Ribo M, Delgado-Mederos R, Santamarina E, Delgado P, Montaner J, Alvarez-Sabín J, Molina CA: Tandem internal carotid artery/middle cerebral artery occlusion: an independent predictor of poor outcome after systemic thrombolysis. Stroke 2006;37:2301-2305.

-4 Lavallée PC, Mazighi M, Saint-Maurice JP, Meseguer E, Abboud H, Klein IF, Houdart E, Amarenco P: Stentassisted endovascular thrombolysis versus intravenous thrombolysis in internal carotid artery dissection with tandem internal carotid and middle cerebral artery occlusion. Stroke 2007;38:22702274.

-5 Baumgartner RW, Georgiadis D, Nedeltchev K, Schroth G, Sarikaya H, Arnold M: Stent-assisted endovascular thrombolysis versus intravenous thrombolysis in internal carotid artery dissection with tandem internal carotid and middle cerebral artery occlusion. Stroke 2008;39:e27-e28.

-6 Pham MH, Rahme RJ, Arnaout O, Hurley MC, Bernstein RA, Batjer HH, Bendok BR: Endovascular stenting of extracranial carotid and vertebral artery dissections: a systematic review of the literature. Neurosurgery 2011;68:856-866.

-7 Becktepe JS, You SJ, Berkefeld J, Neumann-Haefelin T, Singer OC: Clinical outcome after mechanical recanalization as mono- or adjunctive therapy in acute stroke: importance of time to recanalization. Cerebrovasc Dis 2011;32:211-218.

8 Delgado MG, García R, Murias E: Is intravenous thrombolysis useful in carotid artery dissection with tandem occlusion? J Stroke Cerebrovasc Dis DOI: 10.1016/j.jstrokecerebrovasdis.2010.05.003.

9 Pfefferkorn T, Holtmannspoetter M, Patzig M, Brueckmann H, Ottomeyer C, Opherk C, Dichgans M, Fesl G: Preceding intravenous thrombolysis facilitates endovascular mechanical recanalization in large intracranial artery occlusion. Cerebrovasc Dis 2011;31(suppl 2):abstract 8.

10 Shuaib A, Butcher K, Mohammad AA, Saqqur M, Liebeskind DS: Collateral blood vessels in acute ischaemic stroke: a potential therapeutic target. Lancet Neurol 2011;10:909-921.

11 Ribo M, Flores A, Rubiera M, Pagola J, Sargento-Freitas J, Rodriguez-Luna D, Coscojuela P, Maisterra O, Piñeiro S, Romero FJ, Alvarez-Sabin J, Molina CA: Extending the time window for endovascular procedures according to collateral pial circulation. Stroke 2011;42:3465-3469.

12 Miteff F, Levi CR, Bateman GA, Spratt N, McElduff P, Parsons MW: The independent predictive utility of computed tomography angiographic collateral status in acute ischaemic stroke. Brain 2009;132:22312238. 
13 Lekoubou A, Cho TH, Nighoghossian N, Kumako V, Derex L, Trouillas P, Turjman F: Combined intravenous recombinant-tissular plasminogen activator and endovascular treatment of spontaneous occlusive internal carotid dissection with tandem intracranial artery occlusion. Eur Neurol 2010;63:211-214

14 Dabitz R, Triebe S,Leppmeier U, Ochs G, Vorwerk D: Percutaneous Recanalization of Acute Internal Carotid Artery Occlusions in Patients with Severe Stroke. Cardiovasc Intervent Radiol 2007;30:34-41.

15 Hauck EF, Natarajan SK, Ohta H, Ogilvy CS, Hopkins LN, Siddiqui AH, Levy EI: Emergent endovascular recanalization for cervical internal carotid artery occlusion in patients presenting with acute stroke. Neurosurgery 2011;69:899-907. 